Association for Information Systems AIS Electronic Library (AISeL)

Wirtschaftsinformatik Proceedings 2003

Wirtschaftsinformatik

September 2003

\title{
Organisationale Integration von E-Learning in Unternehmen - ein Referenz-Informationsmodell
}

Corinna Haas

Universität Osnabrück, chaas@uos.de

Frederik Ahlemann

Universität Osnabrück

Uwe Hoppe

Universität Osnabrück

Follow this and additional works at: http://aisel.aisnet.org/wi2003

\section{Recommended Citation}

Haas, Corinna; Ahlemann, Frederik; and Hoppe, Uwe, "Organisationale Integration von E-Learning in Unternehmen - ein ReferenzInformationsmodell" (2003). Wirtschaftsinformatik Proceedings 2003. 37.

http://aisel.aisnet.org/wi2003/37

This material is brought to you by the Wirtschaftsinformatik at AIS Electronic Library (AISeL). It has been accepted for inclusion in Wirtschaftsinformatik Proceedings 2003 by an authorized administrator of AIS Electronic Library (AISeL). For more information, please contact elibrary@aisnet.org. 
In: Uhr, Wolfgang, Esswein, Werner \& Schoop, Eric (Hg.) 2003. Wirtschaftsinformatik 2003: Medien - Märkte - Mobilität, 2 Bde. Heidelberg: Physica-Verlag

ISBN: 3-7908-0111-9 (Band 1)

ISBN: 3-7908-0116-X (Band 2)

(C) Physica-Verlag Heidelberg 2003 


\title{
Organisationale Integration von E-Learning in Unternehmen - ein Referenz-Informations- modell
}

\author{
Corinna Haas, Frederik Ahlemann, Uwe Hoppe \\ Universität Osnabrück
}

\begin{abstract}
Zusammenfassung: Um E-Learning dauerhaft in die Aus- und Weiterbildung und in das Informationssystem von Unternehmen integrieren zu können, ist es nicht ausreichend, lediglich didaktische und technologische Ansätze zu berücksichtigen. Vielmehr muss für eine systematische Integration in bestehende Unternehmensstrukturen auch die organisationale Perspektive einbezogen werden. Dieser Beitrag stellt ein Referenz-Informationsmodell vor, welches die Konzeption, Organisation und Umsetzung von E-Learning-Prozessen unterstützen und damit die organisationale Integration des E-Learning wesentlich fördern kann. Es identifiziert für den Integrationsprozess zentrale Aufgaben und Informationsstrukturen und kann dazu beitragen, die bei der Einführung des E-Learning anfallenden Kosten zu reduzieren.
\end{abstract}

Schlüsselworte: E-Learning in Unternehmen, organisationale Integration, betriebliche Aus- und Weiterbildung, Informationssystem, Referenzmodell, Prozesse und Datenstrukturen

\section{Einführung}

Die Pionierphase des E-Learning ist weitgehend abgeschlossen, zwei bedeutende Einsichten sind gewonnen. Die erste Einsicht lautet: Die Entwicklung elektronischer Lehr-Lernangebote darf nicht, wie lange geschehen, allein vom Primat der Technik getragen werden. Die zweite Einsicht lautet: Methodisch-Didaktische Ansätze sind eine tragende Säule bei der Entwicklung von E-Learning-Angeboten.

E-Learning soll Unternehmen die Möglichkeit bieten, die Kosten der Weiterbildung durch kürzere Abwesenheit vom Arbeitsplatz sowie Einsparung von Reisekosten zu minimieren. Just-in Time-Learning soll es dem Arbeitnehmer ermöglichen, aktuelle Wissenslücken individuell und schnell zu schließen. Diese Vorteile, um nur einige Beispiele zu nennen, werden immer wieder mit dem ELearning verbunden (siehe bspw. [LaPä02, S. 17ff.; HaOt02, S. 21ff.; Witt95]). An der systematischen und umfassenden Beantwortung der Frage allerdings, wie 
E-Learning als integrativer Bestandteil in der betrieblichen Aus- und Weiterbildung verankert und als strategische Maßnahme für die Unternehmensentwicklung genutzt werden kann, mangelt es dagegen noch immer ${ }^{1}$. Wie sehen in einem Unternehmen die Schnittstellen von E-Learning und Umsystem aus? Wo findet sich E-Learning im Spannungsfeld von strategischer Unternehmensplanung auf der einen und Personalentwicklung auf der anderen Seite wieder? Wie kann sichergestellt werden, dass E-Learning im Unternehmen tatsächlich Nutzen stiftet? Dies sind Fragen, die nun in der Konsolidierungsphase des E-Learning durch einen organisationalen Bezugsrahmen beantwortet werden können.

Der vorliegende Beitrag stellt sich dieser Herausforderung. Er verfolgt das Ziel, anhand eines von den Autoren modellierten Referenz-Informationsmodells aufzuzeigen, wie E-Learning über eine Verankerung im betrieblichen Planungssystem systematisch in die Aus- und Weiterbildung von Unternehmen integriert werden kann. Das Modell repräsentiert dabei ein aus Fallbeispielen großer Unternehmen abgeleitetes hypothetisches Konstrukt. Eine empirische Überprüfung des Modells in der Praxis steht noch aus, ist aber im Rahmen der weiteren Forschungsarbeit geplant.

Einem deduktiven Argumentationsstrang folgend werden in Kapitel 2 zunächst die Begriffe E-Learning und Referenz-Informationsmodell definiert. Weiterhin werden die dem Modell zu Grunde liegenden Modellierungssprachen kurz erläutert. In Kapitel 3 wird der Makro-Prozess des E-Learning, welcher in Anlehnung an den klassischen Management-Zyklus modelliert wurde, vorgestellt. Der MakroProzess zeigt, wie E-Learning in Organisationen integriert werden kann. Jede Funktion dieses hoch-aggregierten Prozesses wird ihrerseits in spezifischen Mikro-Prozessen und Datenmodellen abgebildet. Dies soll am Beispiel des Datenund Prozess-Modells zur E-Learning-Planung veranschaulicht werden. Der Beitrag schließt mit einer Zusammenfassung und einem kurzen Ausblick auf weitere Forschungsaufgaben (Kapitel 4).

\footnotetext{
Die Auseinandersetzung mit Fragen des E-Learning findet zumeist stark fokussiert statt. Es existieren zahlreiche Arbeiten zu Vorgehensmodellen bei der Entwicklung von E-Learning-Angeboten oder zu den Nutzeffekten des E-Learning usw.. Eine umfassende Analyse der E-Learning-Integration in Unternehmensstrukturen, die insbesondere den klassischen Management-Zyklus auch in Verbindung mit dem Informationsmanagement berücksichtigt, liegt allerdings noch nicht vor.
} 


\section{E-Learning und Modellierung von Referenz- Informationsmodellen: Eine Begriffsklärung}

\section{E-Learning}

Der Begriff E-Learning muss sich gegenüber einer Vielzahl an Begriffen behaupten. Es wird gesprochen von virtuellem Lernen, Online-Lernen, Multimedialem Lernen, (Open)Distance Learning und eben auch E-Learning [vgl. auch Döri02, S. 247f.]. Häufig werden diese Begriffe synonym verwendet, obwohl sie allem Anschein nach nicht das selbe meinen. Dies ist sicherlich auch darauf zurückzuführen, dass die Auseinandersetzung mit dem Thema in einem deutlich interdisziplinären Kontext erfolgt, welcher u.a. aus den Disziplinen (Wirtschafts-)Informatik, Informations- und Wissensmanagement, Pädagogik, Soziologie und Psychologie gebildet wird [HaHo02, S. 88].

Im vorliegenden Beitrag werden unter dem Begriff E-Learning alle Formen des vernetzten Lernens subsumiert. Er wird bewusst nicht gleichgesetzt mit multimedialem Lernen. Denn dieses zielt lediglich auf Lernen mit Systemen ab, welche in der Lage sind, verschiedene Datentypen wie z.B. Texte, Grafiken, Ton und Bewegtbilder [Kerr01, S. 13] und somit diskrete, raumbasierte Medien mit kontinuierlichen, zeitbasierten Medien zu integrieren und zu synchronisieren $\left[\operatorname{Rei}^{+} 98\right.$, S. 22]. Auch die Gleichsetzung mit Virtuellem Lernen erscheint wenig zweckmäßig. Versteht man nämlich Virtualisierung u.a. als ein Konzept zur Leistungssteigerung von Organisationen, kann lediglich von Lernen in Virtuellen Organisationen (Bsp. Virtuelle Universitäten) bzw. Virtuellen Räumen oder Lernumgebungen gesprochen werden [HaHo02, S. 92].

Was bedeutet nun E-Learning genau? Das ,e' steht für electronic. Es geht bei diesem Ansatz um Lernen auf der Basis von elektronischen Verbindungen bzw. Netzen. Nach Rosenberg [Rose01, S. 28] „E-Learning refers to the use of Internet technologies to deliver a broad array of solutions that enhance knowledge and performance". Keating [Keat02, S. 02] dagegen fokussiert E-Learning auf die Anwendung von Informationstechnologie im Lernprozess. Netzwerke wie das Internet macht er hierfür nicht explizit zur Voraussetzung. Vielmehr findet E-Learning nach seinem Verständnis bereits dann statt, wenn ein Lerner eine Software für ein Computer Based Training (CBT) bearbeitet. Ein ähnliches Verständnis skizziert Scholz [Scho01], indem er E-Learning als den Oberbegriff jeglicher Formen elektronisch gestützten Lernens mit dem konstitutiven Element der Computertechnologie beschreibt. Eine sehr ausführliche, um eine Management-Komponente erweiterte Definition liefern Back et al. [Bac ${ }^{+} 01$, S. 36f.]. Die Autoren definieren ELearning als Lernen, das mit Informations- und Kommunikationstechnologien respektive mit darauf aufbauenden (E-Learning-)Systemen unterstützt bzw. ermöglicht wird. Das Spektrum von E-Learning-Systemen reicht nach Back et al. von 
Sprachlernprogrammen auf CD-ROM über webbasierte Kurse mit kollaborativen Räumen und interne oder externe Lern- und Wissensportale mit heterogenen Contents und Plattformen bis hin zu integrierten Systemen, die Kompetenzprofile erheben, individualisierte Kursangebote zusammenstellen, Daten mit ERP-Systemen auswerten und abgleichen sowie an Managementinformationssysteme weitergeben.

Welchen Mehrwert kann E-Learning gegenüber klassischen Weiterbildungsformen bieten [vgl. bspw. Witt95]? Weshalb erscheint es zweckmäßig, E-Learning in die betriebliche Aus- und Weiterbildung zu integrieren? Der zentrale Nutzen ist sicherlich in der verstärkten räumlichen und zeitlichen Flexibilität des Lehrens und Lernens zu sehen. Lehren und Lernen wird an verschiedenen Orten und zu verschiedenen Zeitpunkten möglich. Ein wichtiger und aktuell häufig unter dem Ansatz des Blended Learning diskutierter Aspekt ist weiterhin, dass E-Learning klassischen Präsenzveranstaltungen eine neue Qualität ${ }^{2}$ verleihen kann, indem ELearning und Präsenzlernen in einem Lehr-Lern-Arrangement integriert werden. Singh \& Reed [SiRe01] stellen fest: "Blended learning focuses on optimizing achievement of learning objectives by applying the 'right' learning technologies to match the 'right' personal learning style to transfer the 'right' skills to the 'right' person at the 'right' time." Als Formen der Verknüpfung nennen sie beispielsweise Blending Offline and Online Learning, Blending Self-Paced and Live, Collaborative Learning oder Blending Structured and Unstructured Learning. Sauter \& Sauter [SaSa02] verstehen unter Blended Learning ein integriertes Lernkonzept, welches die verfügbaren Vernetzungsmöglichkeiten über Internet oder Intranet in Verbindung mit klassischen Lernmethoden in einem Lehr-Lernarrangement nutzt. Troha [Troh02] fokussiert die informationstechnische Komponente des Blended Learning auf das Internet, indem er konstatiert: „Blended learning is hot and understandably so, combining the best features of online learning [...] with the best features of classroom instruction (e.g., live, face-to-face, interaction)." Wie auch die angeführten Erklärungsbeispiele resultieren zum Blended Learning gemachte Aussagen zumeist aus dem Kontext der Weiterbildungspraxis [vgl. HoHa03]. Dies unterstreicht die Notwendigkeit einer grundlegenden und detaillierten organisationalen bzw. organisatorischen Betrachtung der Integration des E-Learning in bestehende Weiterbildungscurricula ${ }^{3}$ und -strukturen eines Unternehmens auf der Mikroebene.

Qualität wird in diesem Kontext gemäß der syntaktischen Herleitung verstanden als Güte bzw. Beschaffenheit.

3 Zum Thema Curriculare Integration von E-Learning-Angeboten in bestehende Curricula vgl. [HoHa03]. 


\section{Referenz-Informationsmodelle}

Nach Schütte ist ein Referenz-Informationsmodell definiert als “... das Ergebnis einer Konstruktion eines Modellierers, der für Anwendungssystem- und Organisationsgestalter Informationen über allgemeingültig zu modellierende Elemente eines Systems zu einer Zeit als Empfehlungen mit einer Sprache deklariert, so dass ein Bezugspunkt für ein Informationssystem geschaffen wird.” [Schü98, S. 69; Kursivdruck und Unterstreichungen entfernt]. Referenzmodelle kommen in unterschiedlichen Anwendungsbereichen zum Einsatz, darunter die Softwareentwicklung, die Organisationsgestaltung, das Qualitätsmanagement oder auch das Wissensmanagement [BeSc96, S. 23f.]. Zu den Vorteilen ihrer Anwendung zählen die Kosten- und Risikoreduktion, die Verkürzung von Konzeptions- und Entwicklungszeiten und die Erhöhung von Erlösen [BeSc96, S. 27f.; Schü98, S. 75f.].

Grundsätzlich kommen bei der Entwicklung von Referenzmodellen zwei Klassen von Sprachen zum Einsatz: Sprachen zur Beschreibung von dynamischen Systemaspekten und Sprachen zur Beschreibung von statischen Systemaspekten. Im Bereich der Informationssystemmodellierung unterscheidet man diesbezüglich landläufig zwischen Prozess- und Datenmodellen.

In diesem Beitrag werden zur Konstruktion des Referenz-Informationsmodells Ereignisgesteuerte Prozessketten (EPK) für die Prozessmodellierung und Entity-Relationship-Modelle für die Datenmodellierung verwendet.

\subsubsection{Ereignisgesteuerte Prozessketten (EPK)}

Ereignisgesteuerte Prozessketten werden im wesentlichen aus nur drei Modellelementen konstruiert: Ereignissen, Funktionen und Junktoren [Kel $\left.{ }^{+} 92\right]$. Diese Modellelemente werden durch gestrichelte Pfeile miteinander verbunden, die den zeitlich-sachlogischen Ablauf von Ereignissen und Funktionen verdeutlichen (Kontrollfluss). Funktionen sind dabei aktive Elemente, die das Informationssystem von einem Zustand in einen nachfolgenden Zustand überführen und dabei Informationen verarbeiten. Funktionen repräsentieren Aufgaben, die im Rahmen des zu modellierenden Prozesses auszuführen sind, und werden in einer EPK als Rechteck mit abgerundeten Ecken notiert. Ereignisgesteuerte Prozessketten sind bipartite Graphen; Ereignisse und Funktionen wechseln einander stets ab. Ereignisse sind passive Systemelemente, repräsentieren den Systemzustand zu einem bestimmten Zeitpunkt und werden als Sechsecke notiert. Zur Modellierung von Verzweigungen des Kontrollflusses werden Operatoren verwendet. Diese werden als Kreise notiert und erlauben es, eine Funktion mit mehreren Anschlussereignissen zu verknüpfen oder umgekehrt auf ein Ereignis mehrere Funktionen folgen zu lassen. Die semantische Ausdruckskraft von Junktoren wird durch logische Funktionen erhöht: Junktoren können entweder eine UND-, eine ODER- oder XORVerknüpfung bzw. -Aufteilung des Kontrollflusses darstellen.

\subsubsection{Entity-Relationship-Modelle}

Das Grundkonzept von Entity-Relationship-Modellen basiert auf Entitäten (Entities) und Beziehungen (Relationships) [Chen76]. Erstere repräsentieren Objekte 
der realen Welt, letztere repräsentieren Beziehungen zwischen diesen Objekten. In Entity-Relationship-Modellen werden gleichartige Objekte der Realwelt und ihre Beziehungen zu Entity-Typen und Relationship-Typen zusammengefasst. EntityTypen werden graphisch als Rechtecke notiert, Relationship-Typen als Rauten. Beide werden über einfache Linien miteinander verbunden, die üblicherweise mit so genannten Kardinalitäten versehen werden. Die Kardinalitäten bringen zum Ausdruck, wie häufig ein Entity eines bestimmten Typs eine Beziehung mit einem Entity eines anderen Typs eingehen kann. Hier wird zur Notation der Kardinalitäten die Min-Max-Notation verwendet, bei der jede Kante zwischen einem EntityTyp und einem Relationship-Typ mit zwei Kardinalitäten versehen wird. Die erste Kardinalität ist die Minimalkardinalität und drückt aus, wie oft ein Entity mindestens eine Beziehung mit dem entsprechenden anderen Entity eingehen muss. Die zweite Kardinalität ist die Maximalkardinalität und verdeutlicht, wie oft das Entity maximal eine solche Beziehung eingehen kann [ScSt83, S. 50f.]. Unbegrenzte Kardinalitäten werden durch den Buchstaben ,m' ausgedrückt [Stra95, S. 3.].

In den im folgenden vorgestellten Modellen werden einige Erweiterungen des Standard-Entity-Relationship-Modells verwendet. Relationship-Typen, die eine Beziehung mit weiteren Entity-Typen eingehen, werden Aggregationen genannt und als Rauten eingeschlossen von einem Rechteck gezeichnet.

Generalisierungen beziehungsweise Spezialisierungen (im folgenden wird nur noch von Spezialisierung gesprochen) erlauben die Modellierung von Entity-Typen, die dieselben Objekte auf einem unterschiedlichen Abstraktionsniveau beschreiben [SmSm77, S. 107f.]. Spezialisierungen werden als Dreieck notiert. Der Entity-Typ der höheren Abstraktionsstufe (Super-Typ) wird mit dem Dreieck so verbunden, dass die entsprechende Kante eine Ecke des Dreiecks berührt, die SubTypen werden so verbunden, dass die entsprechenden Kanten die gegenüberliegende Seite des Dreiecks berühren. Spezialisierungen werden mit zwei Buchstaben versehen, die den Typ der Spezialisierung zum Ausdruck bringen. Die Buchstaben ,D'/'ND' stehen für eine disjunkte/nicht disjunkte Spezialisierung. Die Buchstaben ,T'/'P' stehen für eine totale/partielle Spezialisierung [EINa94, S. 618f.; Voss00, S. 98f.].

\section{Ausgewählte Referenz-Prozess- und Datenmodelle}

Auf Grund des Umfangs der Problemdomäne ist es nicht möglich, im Rahmen des vorliegenden Beitrages alle unter dem Gesamtmodell subsumierten Prozess- und Datenmodelle vorzustellen und zu diskutieren. Deshalb sollen hier beispielhaft der Makro-Prozess der E-Learning-Integration sowie der Mikroprozess der E-Learning Planung vorgestellt und beschrieben werden. Diese Prozesse können als exemplarisch für die Referenz-Prozess- und Datenmodelle angesehen werden und sind geeignet, einen ersten Einblick in die Forschungsergebnisse zu geben. 


\subsection{Der Makro-Prozess der E-Learning-Integration}

Der E-Learning Makro-Prozess ist ein Management-Prozess, der alle notwendigen Aktivitäten des Planens, Implementierens und Anwendens von E-Learning in Organisationen widerspiegelt. Die Entwicklung dieses Prozesses basiert auf zwei zentralen Forderungen:

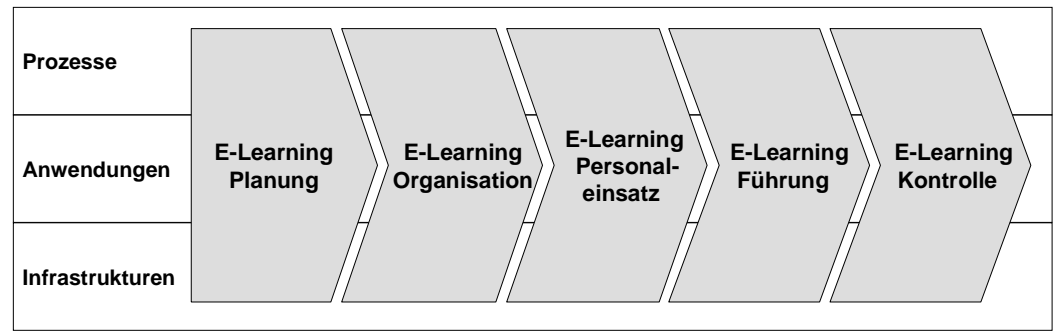

Abbildung 1: Bezugsrahmen der E-Learning-Referenzmodelle

1) Das Management von E-Learning muss alle technischen und organisatorischen Ebenen des E-Learning abdecken (E-Learning Architektur)

Gemäß Wollnik's Referenzmodell für Informationsmanagement kann E-Learning in drei verschiedene Ebenen eingeteilt werden [Woll88]:

a) Die Anwendungsebene des E-Learning: Diese Ebene beinhaltet einerseits Personalentwicklung und -schulung, andererseits aber auch die Nutzung eines E-Learning-Systems, z.B. als Bestandteil des Blended Learning. Technische Aspekte des E-Learning werden auf dieser Ebene nicht behandelt, vielmehr wird E-Learning aus einer prozessualen Perspektive betrachtet.

b) Die Ebene der Entwicklung und Pflege der E-Learning-Inhalte bzw. der Konzeption der E-Learning-Systeme: Auf dieser Ebene ist der Prozess der Konzeption und Umsetzung von E-Learning-Angeboten angesiedelt. Getragen wird dieser Prozess in erster Linie von den Content-Autoren bzw. Trainern, die einerseits Experten für den umzusetzenden Lehrstoff sind, die andererseits aber auch über die notwendigen mediendidaktischen und lernpsychologischen Qualifikationen verfügen. E-Learning-Systeme umfassen nicht allein Software gestützte Lernangebote, sondern auch klassische Lehr-Lernarrangements wie Präsenzseminare.

c) Die Ebene der Bereitstellung und Wartung von E-Learning-Infrastrukturen: Diese Ebene beinhaltet die Entwicklung von E-Learning Softwareplattformen (Lernplattformen), die Installation von Hardware, die Einrichtung von Kommunikationsnetzen (z.B. das Internet) und andere rein technische Aktivitäten. 
Ein adäquates E-Learning-Management berücksichtigt jede der drei Ebenen und setzt ein grundlegendes Verständnis der Abhängigkeiten bzw. Beziehungen zwischen diesen Ebenen voraus.

\section{2) E-Learning bedarf eines vollständigen Management-Prozesses}

Das Management von E-Learning darf nicht auf einzelne, isoliert eingesetzte Managementbausteine reduziert werden. Vielmehr erfordert es eine komplette Managementunterstützung, die von der Planung bis hin zur Kontrolle reicht. Diesem Anspruch folgend, basiert das hier vorgestellte Referenz-Informationsmodell auf dem Managementprozess von Koontz \& O’Donnell [KoDo55]:

a) Planung: E-Learning sollte niemals als Selbstzweck, sondern als eine gut begründete Management-Initiative, die Machbarkeit und Rentabilität berücksichtigt, verstanden werden. ,Ad hoc'-E-Learning-Initiativen sind nicht sinnvoll, da sie Rentabilitätsaspekte nicht in Betracht ziehen.

b) Organisation: Insbesondere dann, wenn E-Learning unternehmensweit praktiziert werden soll, gewinnen organisationale Aspekte an Bedeutung. Spezielle organisatorische Einheiten, die die E-Learning-Infrastrukturen warten und pflegen, E-Learning-Inhalte entwickeln oder einkaufen und die Personalentwicklung und -schulung unterstützen, sind notwendig. Die relevanten Infrastrukturen müssen geschaffen, die Inhalte entwickelt und implementiert werden.

c) Personaleinsatz: Zusätzlich zu dem reinen Aufbau der organisatorischen Einheiten muss die (erstmalige) Stellenbesetzung und die fortlaufende Sicherstellung und Erhalten der Human-Ressourcen erfolgen. Es gilt, ein im optimalen Fall interdisziplinär ausgerichtetes Team zu formen. E-Learning erfordert Mitarbeiter mit sehr unterschiedlichen Fähigkeiten. Die E-Learning Infrastruktur ist hochtechnisiert und verlangt von den MitarbeiterInnen fundiertes Wissen in der Entwicklung bzw. im Umgang mit (E-Learning spezifischen) Hard- und Software-Paketen. Neben den informationstechnischen Kenntnissen und Fähigkeiten werden von den E-Learning-Verantwortlichen aber auch spezifische Kompetenzen u.a. im Bereich der Mediendidaktik erwartet.

d) Führung: Dieser Bereich beinhaltet die Leitung und Feinsteuerung der Personalaus- und -weiterbildung, die durch das E-Learning unterstützt wird. Hier geht es folglich um die Abwicklung der E-Learning-Nutzung im Rahmen der Personalentwicklung.

e) Kontrolle: Die Qualität der E-Learning-Infrastruktur, der E-Learning-Angebote, der organisatorischen Struktur und der mit dem E-Learning verbundenen Geschäftsprozesse muss kontinuierlich evaluiert werden. Die Kontrolle stellt damit den Ausgangspunkt für einen weiteren Managementzyklus dar. 
Der Makro-Prozess des entwickelten Referenz-Informationsmodells berücksichtigt beide oben genannten Bedingungen und integriert die damit zusammenhängenden Konzepte (siehe Abbildung 1).

Wie in Abbildung $2 \mathrm{zu}$ sehen, sollte der Prozess der E-Learning-Integration mit einer preliminary decision in favour of an e-learning initiative, also einer vorläufigen Entscheidung zu Gunsten einer E-Learning-Initiative, die von der obersten Managementebene getroffen wurde, beginnen. Eine Unterstützung durch das TopManagement ist aus Sicht der Autoren unbedingt notwendig, um E-Learning als eine unternehmensweite Initiative durchsetzen zu können. So kann verhindert werden, dass potentielle Synergieeffekte eingebüßt und - diese Gefahr besteht insbesondere in großen, global tätigen Unternehmen - isolierte E-Learning-Anwendungen entwickelt und implementiert werden [Bagu02, S. 288].

E-Learning muss systematisch geplant werden, um dessen Machbarkeit und Rentabilität sicherzustellen (Check E-Learning Feasibility). Dabei können folgende Planungsergebnisse auftreten: (a) Die E-Learning Initiative wird als nicht machbar und unrentabel eingestuft, (b) eine endgültige Entscheidung für die E-LearningInitiative wird getroffen, (c) die Entscheidung wird vertagt, (d) E-Learning wird lediglich punktuell eingesetzt.

Ist die Entscheidung zu Gunsten der E-Learning-Initiative gefallen, muss eine ELearning-Organisationsstruktur aufgebaut werden (Set-up/adapt E-Learning Organization Structure). Dies kann, je nach den Zielen, die mit der E-Learning-Initiative verfolgt werden, zu der Gründung einer neuen organisatorischen Unternehmenseinheit, bspw. einer Corporate University, führen oder sich lediglich in der Neuformulierung von Aufgaben für bereits vorhandene Stellenbeschreibungen niederschlagen.

Ist die Organisationsstruktur für das E-Learning gefunden und realisiert, kann im nächsten Schritt die E-Learning-Infrastruktur (Set-up/adapt Tech. Infrastructure) aufgebaut werden. Wichtig ist dabei, bereits bestehende Personalentwicklungsprozesse zu berücksichtigen. Der Aufbau einer allgemeinen E-Learning-Infrastruktur (technische E-Learning-Plattform, also Learning-Management-Systeme und/oder Content-Management-Systeme) und die Etablierung standardisierter Personalentwicklungs- und Schulungsprozesse sind Vorbedingungen für die Schaffung von Synergieeffekten innerhalb der Organisation. Sie minimieren den Schulungsaufwand, fördern die Transparenz und erlauben der E-Learning-Organisation, ihr gesamtes Potential zu entfalten. Darüber hinaus dient eine gemeinsame E-Learning-Plattform dazu, isolierten E-Learning-Lösungen innerhalb der Organisation vorzubeugen. 


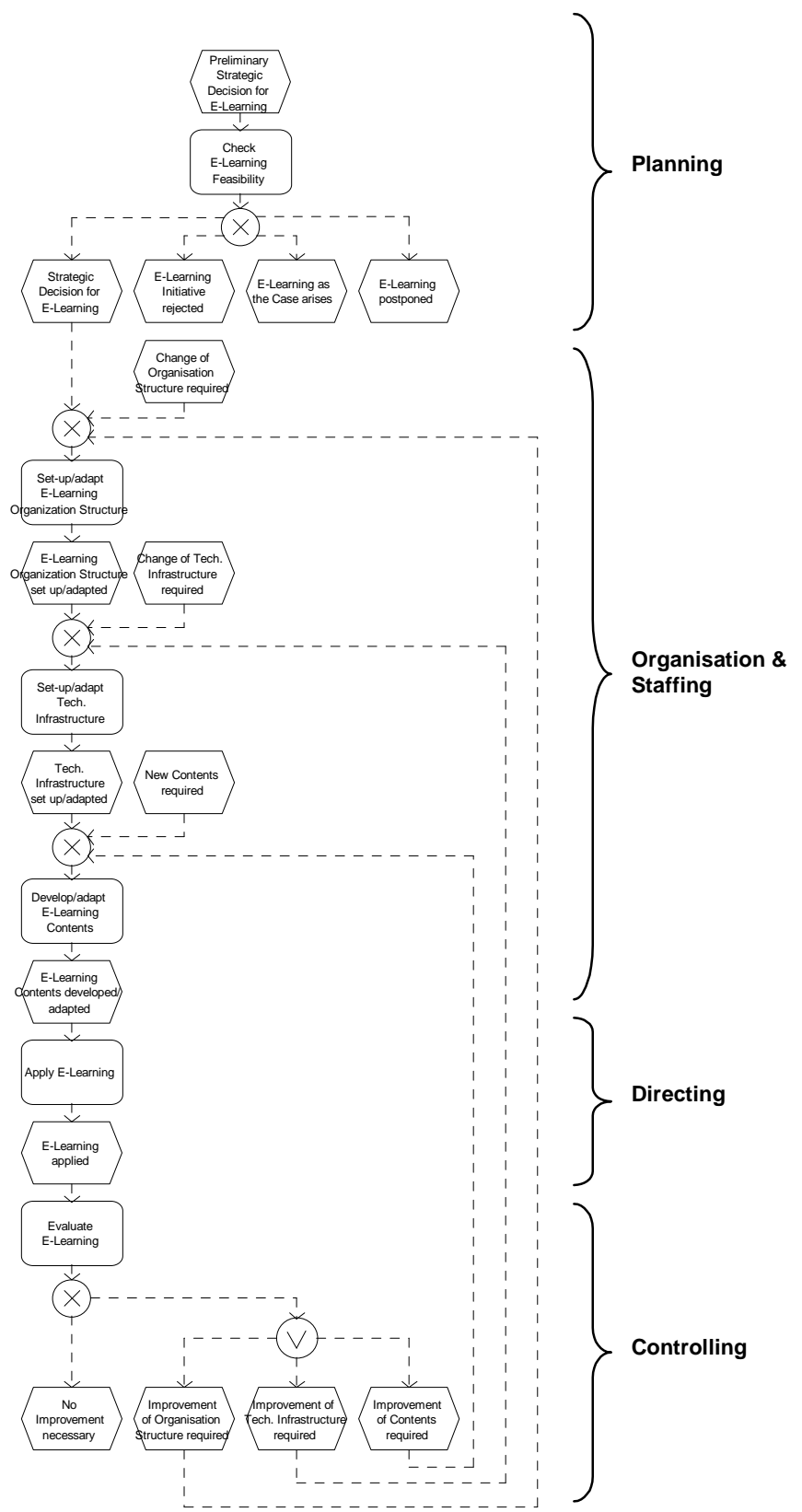

Abbildung 2: Der Makro-Prozess

Sobald die technische und organisatorische Infrastruktur verfügbar ist, können ELearning Inhalte entweder selbst entwickelt und/oder zugekauft werden 
(Develop/adapt E-Learning Contents). Auf Grund des hohen Produktionsaufwandes ist der Einkauf fremd-erstellter Contents bzw. E-Learning-Angebote zwar fast immer kostengünstiger. Allerdings ist der Einsatz dieser Contents oft nur in Bezug auf branchenübergreifende Bildungsbedarfe (bspw. Schulung allgemeiner EDV-Kenntnisse) möglich. Zur Vermittlung unternehmensspezifischer Bildungsinhalte müssen die E-Learning-Angebote inhouse bzw. von damit speziell beauftragten E-Learning-Dienstleistern konzipiert werden.

Sind die E-Learning-Angebote verfügbar, können diese als neuer Baustein in die traditionelle Aus- und Weiterbildung des Unternehmens integriert werden. Dabei ist auch eine Integration im Sinne eines Blended Learning möglich (Apply E-Learning). Zu beachten ist, dass die Einführung von E-Learning-Angeboten aufgrund der hohen Technisierung des Lehr-Lernprozesses von Schulungsmaßnahmen begleitet werden sollte, welche die Adressaten speziell auf das Lernen mit E-Learning-Angeboten vorbereiten (Themen sind u.a. Umgang mit Lehr-Lernplattformen, (Kommunikations-)Dienste des Internet, allgemeine Selbstlernfähigkeit).

Der Management-Prozess endet mit der Kontrolle (Evaluate E-Learning), welche die kontinuierliche Verbesserung der Infrastruktur, der E-Learning-Angebote und der E-Learning-Prozesse sicherstellt. Sie ist der Ausgangspunkt für weitere Prozessiterationen, die zu einer steten Verbesserung des E-Learning innerhalb der Organisation führen. Im Modell wird dies durch die Ereignisse und Funktionen verdeutlicht, die sich an die Evaluation anschließen.

Neben der Mängelbehebung erlauben die zusätzlichen Prozesselemente auch die Anpassung der E-Learning-Infrastruktur, der E-Learning-Prozesse und der ELearning-Inhalte. Dies ist immer dann der Fall, wenn bspw. Bildungsbedarfsanalysen auf zusätzlich nötige Qualifikationen verweisen, neue Geschäftsinitiativen Mitarbeiter mit zusätzlichen Fähigkeiten erfordern, wenn sich Prozesse der Personalentwicklung ändern oder methodisch-didaktische Konzepte einzelner E-Learning-Angebote überarbeitet werden müssen.

\subsection{Mikro-Prozess: E-Learning-Planung}

Der Prozess der E-Learning-Planung ist eine Verfeinerung der ersten Funktion des E-Learning Makro-Prozesses. Er beginnt mit der vorläufigen Entscheidung des Topmanagements zu Gunsten des E-Learning und endet mit der endgültigen unternehmensweiten Entscheidung für die E-Learning Initiative. Dieser Vorgang ist äquivalent zu den Input- und Outputereignissen der korrespondierenden Funktion im Makro-Prozess.

Der Ausgangspunkt des Planungsprozesses ist die vorläufige Entscheidung zu Gunsten einer strategischen E-Learning-Initiative. Solch eine vorläufige Entscheidung basiert typischerweise auf der Erkenntnis, dass E-Learning eine Vielzahl von Vorteilen bieten kann, wie z.B. reduzierte Kosten (Vermeidung von 
Reisekosten, geringe variable Kosten des E-Learning), gestiegende Flexibilität (Lernen zu jeder Zeit, an jedem Ort), kürzere Schulungszeiten (Lernen während und bei der Arbeit an Stelle von Lernen in besonderen Schulungseinheiten, sehr große Zielgruppen können innerhalb kurzer Zeit geschult werden, Schulungen nur dann, wenn aktuell nötig) und verbesserte Schulungsqualität (Verbreitung von , best of breed' Schulungskonzepten, die unabhängig von Trainern stattfinden). Das Topmanagement muss überzeugt werden, dass einige oder alle Vorteile ausgenutzt werden können, denn nur durch dessen Unterstützung kann eine ELearning-Initiative im Unternehmen vorangetrieben werden.

Die vorläufige Entscheidung zu Gunsten von E-Learning mündet meist in der Gründung eines neuen Projektteams, das für die Machbarkeitsstudie verantwortlich ist, die in Verbindung mit der endgültigen Endscheidung das Ergebnis der Planungsphase darstellt. Die Struktur dieser Machbarkeitsstudie kann dem Datenmodell in Abbildung 4 entnommen werden. Detailliert erläutert wird diese Untersuchung auf den folgenden Seiten.

Die erste Aufgabe des Teams ist es, die organisatorischen Einheiten, die potentielle Zielgruppen für E-Learning sein können, zu identifizieren. Dies sind organisatorische Einheiten, deren Stellen von E-Learning in der Zukunft profitieren könnten, weil sie Mitarbeiter mit Fähigkeiten, die durch E-Learning erworben werden können, erfordern. Diese Fähigkeiten sind zum größten Teil kognitiver und weniger motorischer oder sozialer Natur. Es muss berücksichtigt werden, dass die Akzeptanz von E-Learning von Bereich zu Bereich variiert und E-Learning ein Mindestmaß an IT-Kenntnissen erfordert, die nicht unbedingt in jeder organisatorischen Einheit vorhanden sind. Damit ein erster Eindruck über das vorhandende E-Learning-Potential gewonnen werden kann, ist eine Eingrenzung der betroffenen organisatorischen Einheiten notwendig. In dem Datenmodell wird das an Hand der Spezialisierung des Entity Typs Organizational Unit (OU) in zwei Untertypen $O U$ affected by E-Learning und $O U$ not affected by E-Learning dargestellt.

Aus den identifizierten Einheiten können unterschiedliche Zielgruppen abgeleitet werden. Die Zielgruppenbildung orientiert sich an den spezifischen in den Organisationseinheiten benötigten Qualifikationen. Diese können durch Analyse aktueller Stellenbeschreibungen und bestehender Trainingsprogramme oder durch Bildungsbedarfsanalysen, die sich an den zukünftigen Markterfordernissen orientieren, erhoben werden. Die Zielgruppen werden dann so gebildet, dass unabhängig von der organisatorischen Zugehörigkeit Stellen mit gleichen Anforderungen zu einer Gruppe zusammengefasst werden. Bei der Zusammensetzung der Zielgruppen sollten zudem die folgende Aspekte berücksichtigt werden: Die Managementebene, IT-Kenntnisse, bestehende E-Learning Erfahrung sowie vorhandenes (Fach-) Wissen.

Ein kritischer Erfolgsfaktor in diesem Schritt ist die Einbeziehung der betroffenen Zielgruppen und deren Vorgesetzten. Die Akzeptanz von E-Learning wird entscheidend durch die Integration der späteren Adressaten in den Planungsprozess 
beeinflusst. Die frühzeitige Information der Zielgruppen vermittelt diesen das Gefühl, dass ihre Meinung und ihre Wünsche bei der Realisierung der E-LearningAngebote berücksichtigt werden und reduziert Widerstände und Ängste. Einzelinterviews und Gruppendiskussionen sowie Workshops können hierfür die Methode der Wahl sein. In dem Datenmodell wird die Zielgruppendefinition mit Hilfe eines eigenen Entity-Typen dargestellt. Dieser ist mit dem Qualification EntityTyp verbunden, um zum Ausdruck zu bringen, auf welche Qualifikationen sich die Zielgruppendefinition bezieht. Qualifikationen können in einer Hierarchie angeordnet werden, um Qualifikationsdefinitionen auf unterschiedlichen Abstraktionsebenen beschreiben zu können. Qualifikationen befähigen Mitarbeiter, geschäftliche Tätigkeiten (Entity-Typ Activity), die an eine Stelle gebunden sind, auszuüben. Stellen (Entity-Typ $J o b$ ) werden als Spezialisierung des Entity-Typen Organizational Unit angesehen - neben den Abteilungen (Entity-Typ Department).

Die Zielgruppendefinitionen sind wesentlicher Input für den nächsten Prozessschritt. In diesem werden die so genannten E-Learning-Konzepte (E-Learning Concept) für jede dieser Zielgruppen und ihren dazugehörigen Qualifikationen entwickelt. Das E-Learning-Konzept legt die grundlegenden Bedingungen des ELearning, wie z.B. den Lernort, die Sozialform, die Modalitäten der Kontrolle und Überwachung, etc. fest. Es beinhaltet alle Informationen, die für die Diskussion der E-Learning-Initiative im Betriebsrat relevant sind. Diese Diskussion ist nötig, da solch eine Initiative die grundlegenden Rechte, die allgemeinen Arbeitsbedingungen und auch die Vergütung bzw. Entlohnung der Mitarbeiter betrifft. Zu einem späteren Zeitpunkt umfasst das E-Learning-Konzept dann auch Hinweise zu notwendigen Veränderungen von Geschäftsprozessen, die mit E-Learning in Beziehung stehen. Betroffen sind z.B. Prozesse zur Versorgung der Mitarbeiter mit der notwendigen Software. In dem Datenmodell besitzt das E-Learning-Konzept einen eigenen Entity-Typ, der mehrere Spezialisierungen aufweist, die die möglichen Ausprägungen eines E-Learning-Konzeptes andeuten. Der Relationstyp Concept Assignment ermöglicht die Verbingung solcher Konzepte zu den Target Group Qualification Assignments. Dies zeigt, dass jeder Qualifikation, die eine Zielgruppe erwerben muss, ein Konzept zugeordnet wird. 


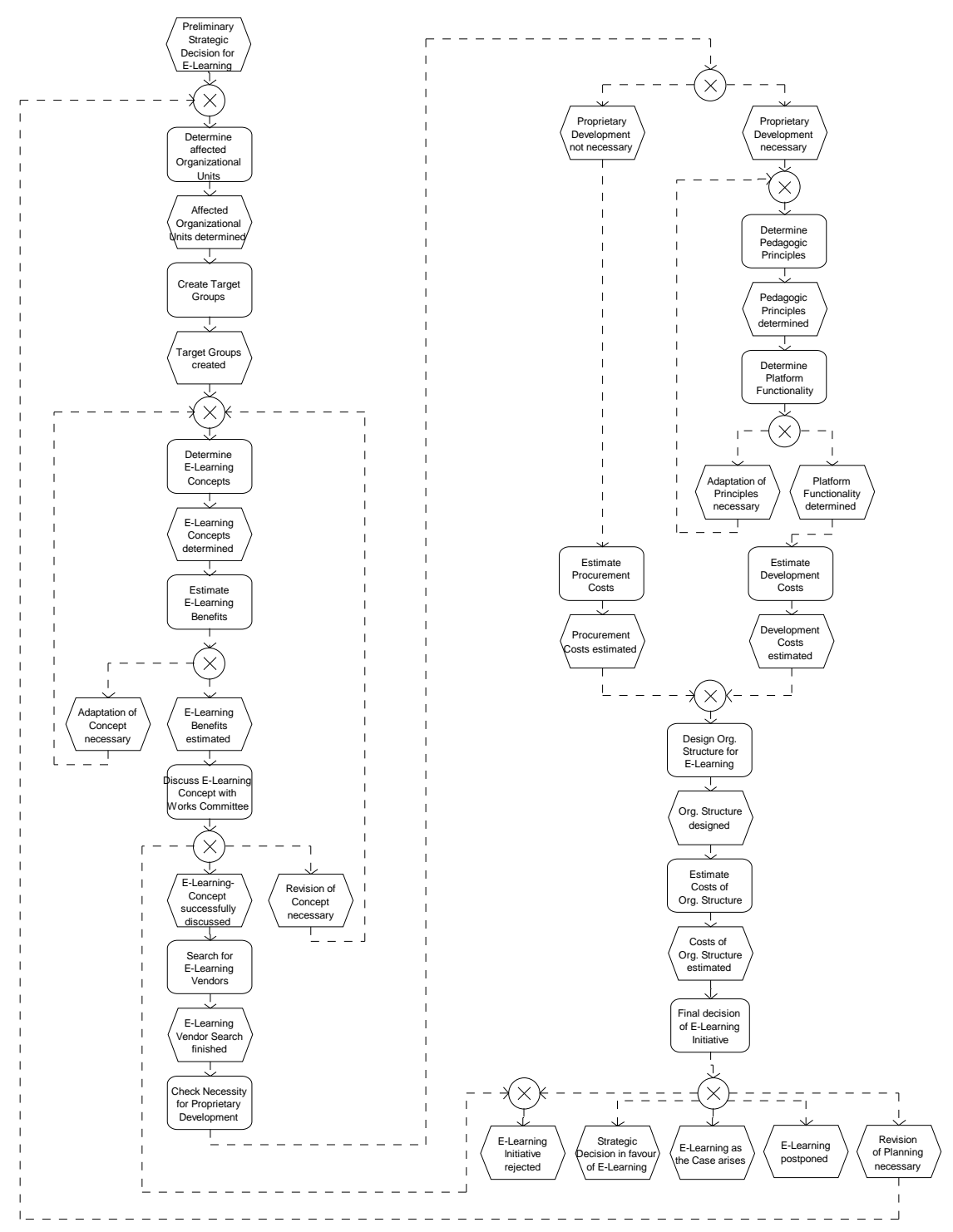

Abbildung 3: Der Planungsprozess des E-Learning

Die E-Learning-Konzeptdefinitionen stellen eine gute Grundlage für die Analyse der Vorteilhaftigkeit des E-Learnings dar. Im Allgemeinen besteht dabei ein Bewertungsproblem, da die monetären Auswirkungen des E-Learning aufgrund vielfältiger Interdependenzen $\mathrm{zu}$ anderen Unternehmensbereichen nicht direkt bestimmt werden kann, eine isolierte Betrachtung ist also nicht möglich. Folglich basiert eine Wirtschaftlichkeitsanalyse immer auf plausiblen Schätzungen, die An- 
nahmen erfordern. Solche Schätzungen können zu der Erkenntnis führen, dass die Vorteile von E-Learning zu gering sind, um eine Realisation des/r Konzeptes/e zu rechtfertigen. In diesen Fällen ist eine Schleife im Managementprozess vorgesehen. Diese ermöglicht eine Anpassung bzw. eine Weiterentwicklung des Konzeptes.

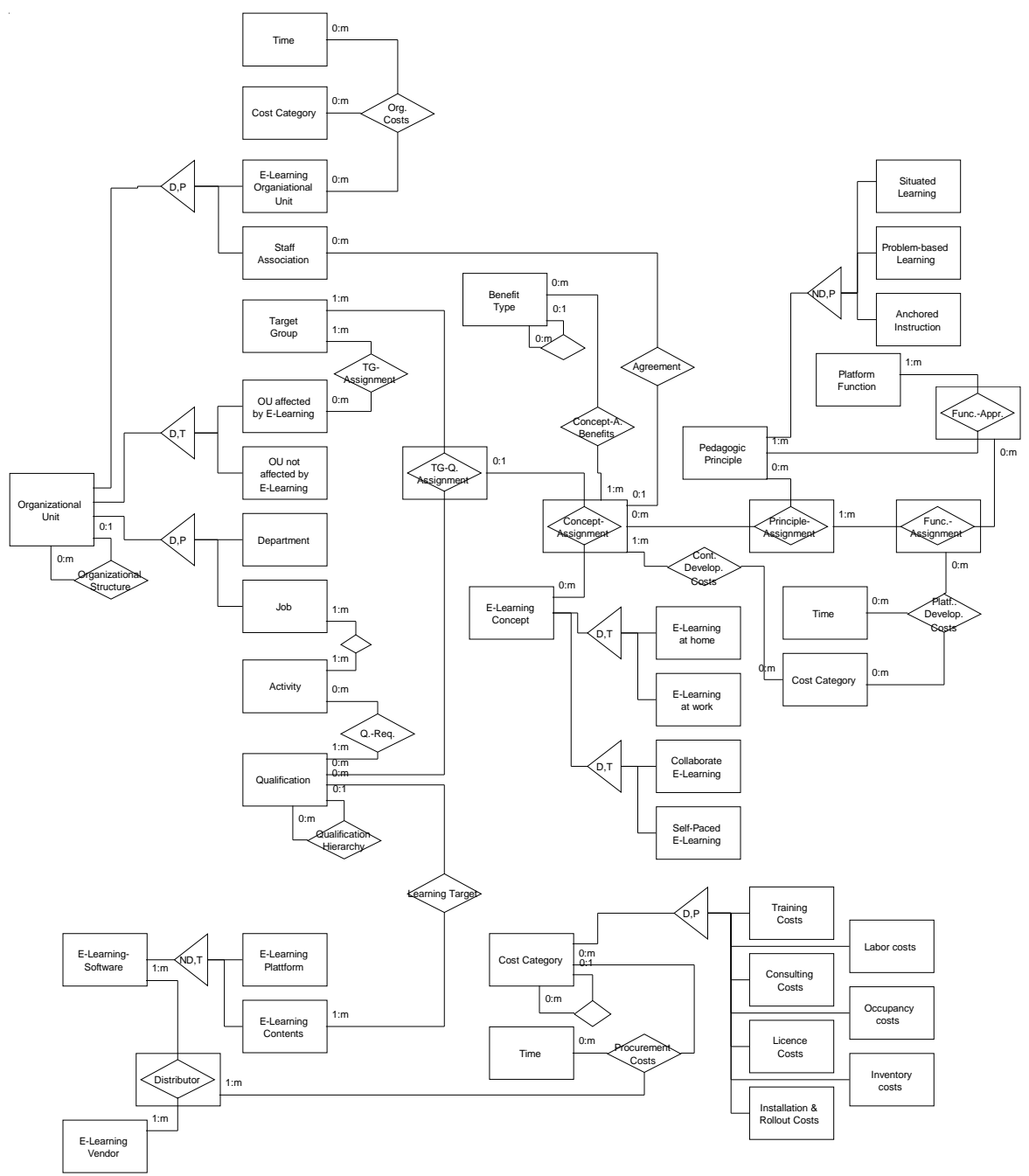

Abbildung 4: Datenmodell zur Planung des E-Learning

Im Datenmodell wird die Wirtschaftlichkeitsanalyse durch den uminterpretierten Relationshiptypen Concept-Assignment und dem Relationshiptypen Concept-A. Benefits, der es ermöglicht, den Nutzen für jedes Concept-Assignment abzubilden, repräsentiert. 
E-Learning-Konzepte und -Vorteile werden mit dem Betriebsrat diskutiert, wenn gesetzliche Bestimmungen eine solche Abstimmung erforderlich machen. Diese Abstimmung wird durch die nächste Funktion in dem Prozessmodell repräsentiert. Das Ziel ist hier, zu einer gegenseitigen Vereinbarung über den Nutzen von ELearning innerhalb des Unternehmens zu gelangen. Wenn eine solche Vereinbarung nicht erreicht werden kann, ist eine Wiederholung vorgesehen und die Ergebnisse dieser Diskussion werden genutzt, um das/die E-Learning Konzept/e anzupassen und zu modifizieren. Besonders in Deutschland hat der Betriebsrat ein weitreichendes Mitspracherecht bezüglich Schulungen, Arbeitsbedingung und Arbeitszeiten. In dem Datenmodell ist der Betriebsrat als eine spezielle Organisationseinheit modelliert, die mit dem Entity-Typen Concept Assignment verbunden ist, um zu verdeutlichen, dass eine Vereinbarung besteht.

Im nächsten Schritt werden die verabschiedeten E-Learning Konzepte und die Zielgruppenbeschreibungen genutzt, um am Markt bereits verfügbare E-LearningAngebote ausfindig zu machen. Auf der einen Seite geht es darum, eine E-Learning-Plattform, über die die E-Learning-Angebote distribuiert werden sollen, auszuwählen ${ }^{4}$. Auf der anderen Seite müssen evt. bereits am Markt verfügbare bedarfsgerechte Contents evaluiert werden. Es gilt eine Entscheidung für den Einkauf der verfügbaren Angebote bzw. für eine proprietäre Software-Entwicklung zu treffen.

Insbesondere große Unternehmen weisen meist sehr spezielle Schulungsbedarfe auf, die den Einsatz von Standardsoftware verbieten. Eine Marktanalyse kann in diesem Falle dafür genutzt werden, die modernste (state-of-the-art) Software ausfindig zu machen und von der Standardsoftware ,zu lernen'. Kleine und mittelgroße Unternehmen dagegen müssen i.d.R. die im Rahmen der Marktanalyse evaluierte Standardsoftware einkaufen. Für sie wäre eine proprietäre Softwareentwicklung nicht profitabel. ${ }^{5}$ Im Datenmodell sind die Softwareanbieter mit Hilfe des Entity-Typs E-Learning Vendor dargestellt. Die Software selber besitzt einen eigenen Entity-Typ (E-Learning Software). Da mehrere Anbieter die gleiche Software verkaufen können, sind beide Entity-Typen mit dem Relationstyp Distributor verbunden.

Der Entity-Typ E-Learning Contents ist mit dem Entity Typ Qualification verbunden, um zu verdeutlichen, dass eine Software stets dem Erwerb einer spezifischen Qualifikation dient.

4 Zum Auswahlprozess und den Bewertungskriterien von Lernplattformen vgl. u.a. $\left[\mathrm{Baum}^{+}\right.$02; Schu03].

5 Nach Hasebrook \& Otte [HaOt02, S. 24] sind Investitionen in die Entwicklung von ELearning-Angeboten erst ab einer Nutzerzahl von 100 bis 200 NutzerInnen bzw. bei einem längerfristigen Einsatz der Angebote rentabel. Witte [Witt95, S. 168] stellt fest, dass eine Computer Aided Instruction (CAI) ab einer Lernerpopulation von mindestens 200 Personen kostengünstiger sei als alternative personelle Bildungsmaßnahmen. 
Ist eine proprietäre Softwareentwicklung erforderlich, sind die bereits entwickelten Rahmenkonzepte des E-Learning noch einmal zu verfeinern. Nur so ist es möglich, eine sinnvolle und realistische Schätzung des Entwicklungsaufwandes abzugeben. Ein bedeutender Kostentreiber sind bsp. die evtl umfangreichen Funktionalitäten der E-Learning Software, die u.a. aus den pädagogischen Prinzipien, auf denen die Software basiert, abgeleitet werden können. In dem Datenmodell ist dies mit dem Enitity-Typ Pedagogic Principle und dessen Spezialisierungen dargestellt. Der Entity-Typ Platform Functionality ist mit Pedagogic Principle verbunden, um zu verdeutlichen, dass bestimmte Plattformen-Funktionalitäten spezifische pädagogische Prinzipien unterstützen. Pedagogic Principles können den Concept Assignments zugeordnet werden. Damit wird ein Brückenschlag zwischen den Funktionalitäten der Lernplattform und dem E-Learning-Konzept unterstützt. Dies unterstreicht die notwendigen Wechselwirkungen zwischen ELearning-Konzept, pädagogischen Prinzipien bzw. Paradigmen und Softwarefunktionalitäten.

Die weiteren Bausteine der ereignisgesteuerten Prozesskette fokussieren Kostenschätzungen. Wird eine E-Learning-Software zugekauft, müssen die dabei voraussichtlich entstehenden Kosten geschätzt werden. Im Datenmodell wird dies durch den Relationstyp Procurement Costs ausgedrückt. Anschaffungskosten haben immer bestimmte Kostenkategorien und erstrecken sich über einen bestimmten Zeitraum. Die Spezialisierungen der Kostenkategorien stehen beispielhaft für typische Anschaffungskosten wie Schulung, Beratung und Lizenzgebühren.

Hat man sich für eine proprietäre Softwareentwicklung entschieden, müssen die dazugehörigen Kosten für die Plattform und die Entwicklung geschätzt werden. Aus diesem Grund sind die korrespondierenden Entity-Typen mit Function Assignment (Plattformkosten) und dem Concept Assignment (inhaltliche Kosten) verbunden.

Im nächsten Schritt kann die organisatorische Struktur der E-Learning-Organisation entworfen werden. In kleinen und mittelgroßen Unternehmen wird sich die E-Learning-Initiative typischerweise in ausführlicheren Stellenbeschreibungen in der Personal- und der IT-Abteilung niederschlagen. Großunternehmen dagegen haben häufig spezielle Anforderungen, die dazu führen, dass gesonderte Unternehmenseinheiten oder auch Tochterunternehmen entstehen. Im Datenmodell repräsentiert die E-Learning Organizational Unit eine Spezialisierung des EntityTyps Organizational Unit.

Ist die organisatorische Struktur geplant, können die daraus resultierenden Kosten geschätzt werden. Diese setzen sich zum größten Teil aus Lohnkosten zusammen. Ergänzt werden sie, insbesondere in der Anfangsphase der E-Learning-Initiative, durch Kosten für die Geschäftsausstattung und die technische Ausstattung. I.d.R. sind die Inbetriebsetzungskosten deutlich höher anzusetzen als die anschließenden Kosten für den Dauerbetrieb der Infrastruktur. 
Basierend auf den in den oben skizzierten Planungsschritten gewonnenen Erkenntnissen kann nun die endgültige Entscheidung zu Gunsten oder gegen die unternehmensweite E-Learning-Initiative getroffen werden. Im Ergebnis wird das Topmanagement entweder (1) sein Interesse an der E-Learning-Initiative bekunden und sie starten, (2) die Initiative ablehnen, (3) Verfeinerungen und Anpassungen verlangen (die zu einem wiederholten Durchlauf durch die Prozesskette führen), (4) die Initiative verschieben oder (5) keine unternehmensweite Initiative starten, sondern andere Managementebenen darum bitten, den Umständen entsprechend zu entscheiden, ob E-Learning eingeführt werden soll oder nicht.

\section{Zusammenfassung und Ausblick}

Um E-Learning dauerhaft in die Aus- und Weiterbildung und das Informationssystem von Unternehmen integrieren zu können, ist es nicht ausreichend, lediglich didaktische und technologische Ansätze zu berücksichtigen. Vielmehr muss für eine systematische Integration in bestehende Unternehmensstrukturen auch die organisationale Perspektive berücksichtigt werden. Das vorgestellte Referenz-Informationsmodell stellt einen ersten Ansatz einer strukturierten Integration des ELearning in das System ,Unternehmen' dar. Auf der Basis eines hypothetischen Konstruktes, welches basierend auf der Analyse ausgewählter Fallstudien entwickelt worden ist, wurde aufgezeigt, welche Phasen und Prozesse eine systematische und effiziente Integration des E-Learning in Unternehmensstrukturen kennzeichnen. Eine wissenschaftliche Validierung und Kompletierung des Modells muss in weiteren Forschungsarbeiten vorgenommen werden. Eine empirische Überprüfung des Modells sowie die Modellierung weiterer Mikro-Prozesse ist vorgesehen.

\section{Literatur}

[Bac $\left.{ }^{+} 01\right]$ Back, A.; Bendel, O.; Stoller-Schai, D.: E-Learning im Unternehmen: Grundlagen, Strategien, Methoden, Technologien. Orell Füssli Verlag AG: Zurich, 2001.

[Bagu02] Bagusat, M.: Das Allianz Lern Forum - E-Learning in der betrieblichen Ausbildung. In: Dittler, U. (Hrsg.): E-Learning: Erfolgsfaktoren und Einsatzkonzepte mit interaktiven Medien. Oldenbourg: München, 2002, S. 283-295.

[Bau 02] Baumgartner, P.; Häfele, H.; Maier-Häfele, K.: E-Learning Praxishandbuch. Auswahl von Lernplattformen. Studien-Verlag: Innsbruck et al., 2002.

[BeSc96] Becker, J.; Schütte, R.: Handelsinformationssysteme. Verlag Moderne Industrie: Landsberg/Lech, 1996. 
[Chen76] Chen, P.P.: The Entity Relationship Model. Toward a Unified View of Data. In: ACM Transactions on Database Systems, 1 (1), 1976, S. 9-36.

[Döri02] Döring, N.: Online-Lernen. In: Issing, L.J.; Klimsa, P. (Hrsg.): Information und Lernen mit Multimedia und Internet. Lehrbuch für Studium und Praxis. 3., vollständig überarbeitete Auflage, Beltz: Weinheim, 2002, S. 247 - 264.

[ElNa94] Elmasri, R.; Navathe, S.B.: Fundamentals of Database Systems. 2. Auflage, Benjamin-Cummings Publishing Co., Inc.: Redwood City, 1994.

[HaHo02] Haas, C.; Hoppe, U.: eLearning für die Zukunft - Begriffe, Erscheinungsformen und Aufgabenfelder. In: Riekhof, H.-C.; Schüle, H. (Hrsg.): E-Learning in der Praxis: Strategien - Konzepte - Fallstudien. Gabler: Wiesbaden, 2002.

[HaOt02] Hasebrook, J.; Otte, M.: E-Learning im Zeitalter des E-Commerce: Die dritte Welle. Huber: Bern et.al., 2002.

[HoHa03] Hoppe, U.; Haas, C.: IMPULS ${ }^{\mathrm{EC}}$ : Ein elektronischer Lehrgang zum Electronic Commerce. Lehrstoff, Einsatzszenarien und curriculare Integration. Erscheint im Tagungsband zum E-Learning Workshop der GOR, Paderborn, 2003.

[Hopp02] Hoppe, U.: Electronic Business und Electronic Commerce - ein Beitrag zur Begriffsbildung. In: Gabriel, R.; Hoppe, U. (Hrsg.). Electronic Business - Theoretische Aspekte und Anwendungen in der betrieblichen Praxis. Physica Verlag: Heidelberg, 2002.

[Keat02] Keating, M.: Geschäftsmodelle für Bildungsportale - Einsichten in den US-amerikanischen Markt. In: Bentlage, U. et al. (Hrsg.): E-Learning. Verlag Bertelsmannstiftung: Gütersloh, 2002.

$\left[\mathrm{Kel}^{+} 92\right]$ Keller, G. et al.: Semantische Prozessmodellierung auf der Grundlage „Ereignisgesteuerter Prozessketten (EPK)“. Veröffentlichungen des Instituts für Wirtschaftsinformatik. Heft 89. Scheer, A.W. (Hrsg.), Saarbrücken, 1992.

[Kerr01] Kerres, M.: Multimediale und telemediale Lernumgebungen: Konzeption und Entwicklung. 2. überarb. Auflage, Oldenbourg: München, 2001.

[KoDo55] Koontz, H.; O’Donnell, C.: Principles of Management: An Analysis of Managerial Functions. McGraw-Hill: New York et al., 1955.

[LaPä02] Lang, M.; Pätzold, G.: Multimedia in der Aus- und Weiterbildung. Grundlagen und Fallstudien zum netzbasierten Lernen. Deutscher Wirtschaftsdienst: Köln, 2002.

[Rei ${ }^{+} 98$ ] Reichwald, R.; Möslein, K.; Sachenbacher, H.; Englberger, H.; Oldenburg, S.: Telekooperation. Verteilte Arbeits- und Organisationsformen. Springer: Berlin et.al., 1998.

[Rose01] Rosenberg, M.J.: E-Learning. Strategies for Delivering Knowledge in the Digital Age. McGraw-Hill: New York et al., 2001.

[SaSa02] Sauter, A. ; Sauter, W. : Blended Learning. Effiziente Integration von E-Learning und Präsenztraining. Luchterhand: Neuwied et al., 2002.

[Scho01] Scholz, C.: E-Learning. In: WiSt - Wirtschaftswissenschaftliches Studium, Zeitschrift für Ausbildung und Hochschulkontakt. Heft 11 2001, Frankfurt/Main. 
[Schu03] Schulmeister, R.: Lernplattformen für das virtuelle Lernen: Evaluation und Didaktik. Oldenbourg: München, 2003.

[Schü98] Schütte, R.: Grundsätze ordnungsmäßiger Referenzmodellierung. Konstruktion konfigurations- und anpassungsorientierter Modelle. Gabler: Wiesbaden, 1998.

[ScSt83] Schlageter, G.; Stucky, W.: Datenbanksysteme. Konzepte und Modelle. 2. Auflage. Teubner: Stuttgart, 1983.

[SiRe02] Singh, H.; Reed, C.: A White Paper: Achieving Success with Blended Learning. URL: www.centra.com/download/whitepapers/ blendedlearning.pdf [12/19/2002].

[SmSm77] Smith, J.M.; Smith, D.C.P.: Database Abstraction - Aggregation and Generalization. In: ACM Transactions on Database Systems. 2 (2), 1977, S. 105-133.

[Stra95] Strahringer, S.: Eine kardinalitätsbezogene Erweiterung der Entity-RelationshipModellierung. Schriften zur Quantitativen Betriebswirtschaftslehre. Nr. 8/95. Technische Hochschule Darmstadt: Darmstadt, 1995.

[Troh02] Troha, F.J.: Bulletproof Instructional Design: A Model of Blended Learning. In: USDLA Journal. 16 (5), 2002. URL: http://www.usdla.org/html/journal/MAY02_Issue/article03.html [12/19/2002].

[Voss00] Vossen, G. : Datenmodelle, Datenbanksprachen und Datenbankmanagementsysteme. 4. Auflage. Oldenbourg: München, 2000.

[Witt95] Witte, K.-H.: Nutzeffekte des Einsatzes und Kosten der Entwicklung von Teachware. Empirische Untersuchung und Übertragung der Ergebnisse auf den praktischen Entwicklungsprozess, Göttingen, 1995.

[Woll88] Wollnik, M.: Ein Referenzmodell des Informations-Management. In: Information Management. (3) 3, 1988, S. 34-43. 